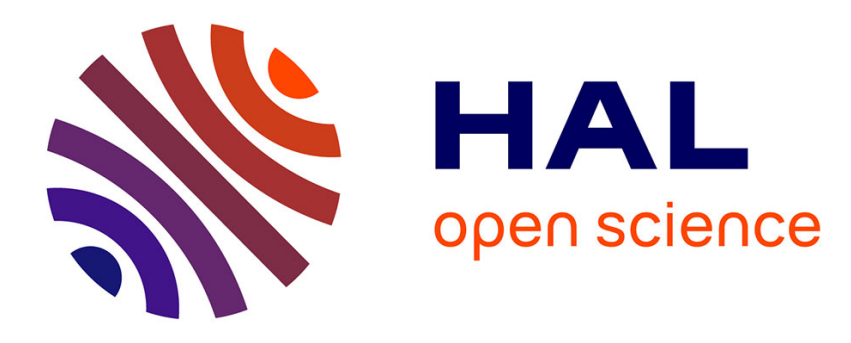

\title{
Students' reasons for introducing auxiliary lines in proving situations
}

Alik Palatnik, Tommy Dreyfus

\section{To cite this version:}

Alik Palatnik, Tommy Dreyfus. Students' reasons for introducing auxiliary lines in proving situations. Eleventh Congress of the European Society for Research in Mathematics Education, Utrecht University, Feb 2019, Utrecht, Netherlands. hal-02402251

\section{HAL Id: hal-02402251 \\ https://hal.science/hal-02402251}

Submitted on 10 Dec 2019

HAL is a multi-disciplinary open access archive for the deposit and dissemination of scientific research documents, whether they are published or not. The documents may come from teaching and research institutions in France or abroad, or from public or private research centers.
L'archive ouverte pluridisciplinaire HAL, est destinée au dépôt et à la diffusion de documents scientifiques de niveau recherche, publiés ou non, émanant des établissements d'enseignement et de recherche français ou étrangers, des laboratoires publics ou privés. 


\title{
Students' reasons for introducing auxiliary lines in proving situations
}

\author{
$\underline{\text { Alik Palatnik }}^{1}$ and Tommy Dreyfus ${ }^{2}$ \\ ${ }^{1}$ Shaanan Academic Religious Teachers' College, Haifa, Israel; umapalatnik@gmail.com \\ ${ }^{2}$ Tel Aviv University, Israel; tommyd@ tauex.tau.ac.il
}

We study proving situations in geometry where students introduce auxiliary lines. We examine students' reasons for introducing auxiliary lines. The overarching theme of the tasks proposed to the students is the comparison of areas of triangles and/or parallelograms. The students gave a wide spectrum of reasons when introducing auxiliary lines. Two main groups of reasons have been discerned for modifying a given situation by introducing auxiliary lines: recalling a learned procedure using some known results or definitions, and anticipating more information from the modified situation. We present a case study of a pair of students who combine reasons of recalling and anticipating nature when introducing auxiliary lines.

Keywords: High school geometry, proof, auxiliary lines, reasoning, task-based interviews.

\section{Introduction}

The objective of this study is to contribute to research on proving in geometry. Specifically, we investigate the introduction of auxiliary elements by students while proving and characterize reasons students give for their actions. Our interest in this process stems from the following facts: First, proving in geometry is difficult for students in general. Second, the introduction of auxiliary lines contributes substantially to students' difficulty with proofs in geometry (e.g. Hsu, \& Silver, 2014; Senk, 1985). Our focus on students' reasons for introducing auxiliary elements led us to build on the seminal work of Pólya (1957), who highlighted the role of auxiliary lines in problem solving.

\section{Theoretical background}

It is widely recognized that in secondary school, proof and proving are still mostly taught in the area of geometry (Herbst, 2004; Fujita \& Jones, 2014). According to Battista (2007) school geometry presents students with a complex network of concepts, representations and ways of reasoning "...that is used to conceptualize and analyze physical and imagined spatial environments" (p. 843). Combining deductive and spatial reasoning is pertinent to proving activities in geometry. Indeed, in his study of American geometry classes, Herbst (2004) demonstrated, among others, a generative type of students' interaction with diagrams in proving activities. Such interactions are characterized by deductive reasoning interrelated with modification of a given diagram. Such interactions are virtually absent from geometry classes, as studies of Japanese secondary schools confirm (Miyazaki, Fujita, \& Jones, 2015) but are central for proofs that require the introduction of an auxiliary element.

According to Pólya (1957), "an element that we introduce in the hope that it will further the solution is called an auxiliary element" (p. 46). In our study, a slightly expanded version of this definition is used. Namely, we consider also the modification of an already existing element as an auxiliary element (see Task 1 in the Methodology section). Moreover, an auxiliary element may be only imagined rather than physically drawn. 
According to Senk (1985) the introduction of auxiliary lines is a critical part in the solution of geometric proof problems. She concluded that the difficulty with auxiliary lines "exemplifies the need to teach students how, why, and when they can transform a diagram in a proof" (p. 455). Ding and Jones (2006) also identified the introduction of an auxiliary line as a source of great difficulty. Hsu (2007) claims that this difficulty is caused by students' need to perceive the diagrams dynamically and apply transformational observation to visualize a solution achievable with auxiliary lines.

Students' difficulty with introducing auxiliary lines when proving can be partially explained by a form of instruction, which does not encourage students to add new lines to the given diagram. For instance, students in the study of Herbst and Brach (2006) self-reported that they expect to play a passive role in interaction with diagrams when proving: They believe that they are usually not expected to introduce auxiliary lines, and if so, they expect to receive a hint to do so from the teacher. This difficulty may be overcome by the incorporation of various types of exploration in proving which makes the introduction of auxiliary lines more transparent (e.g. Fan, Qi, Liu, Wang and Lin, 2017).

Considering these studies and Pólya's fundamental work, it makes sense to consider the introduction of auxiliary lines through the prism of reasons that led to their introduction. Pólya (1957) lists three main reasons for introducing auxiliary lines (p. 46):

1) Trying to use known results;

2) Going back to definitions;

3) Expecting to make the original problem "fuller, more suggestive, more familiar".

Pólya makes a distinction between the first two reasons and the third one. He states that in the third case "we scarcely know yet explicitly how we shall be able to use the elements added. We may just feel that it is a 'bright idea' to conceive the problem that way with such and such elements added" (ibid.). In our study we sought both, to obtain descriptive reports about students introducing auxiliary elements in proving situations, and to identify the types of reasons they provide for introducing them. We adapt Pólya's classification to our aims by introducing the terms recalling (Pólya 1,2) and anticipating (Pólya 3) reasons.

Given this background, our research aims to answer the following questions:

A. What reasons do the students give when introducing an auxiliary line in a proving situation?

B. To which type do these reasons belong, recalling or anticipating?

\section{Methodology}

\section{Students and Tasks}

The participants in our study were high school students studying geometry in Grade 10 - the first grade of Israeli high school. Eighteen students were nominated by their mathematics teachers; ten of them volunteered for the study. The students were grouped into five pairs, following Schoenfeld's (2000) suggestion that students working in pairs will show reliable and natural behavior. 
We sought to create tasks where students would be inclined to draw auxiliary lines in order to make progress. We anticipated that tasks with multiple solutions, each based on different auxiliary lines, will elicit a variety of reasons for introducing auxiliary lines. The topic of areas was suitable as it was already known to the students, but proofs on areas had not been taught in class at the time of the intervention. We used two tasks with different levels of complexity.

Task 1: Quadrilateral ABCD is a parallelogram. Where on side $\mathrm{AB}$ do you have to place point $\mathrm{E}$, so that the area of triangle DEC is a half of the area of the given parallelogram ABCD? Explain your reasoning.

There are multiple ways to show that E can be anywhere on side AB. Figure 1 hints at some of them, highlighting the role of appropriate auxiliary lines in the proofs.

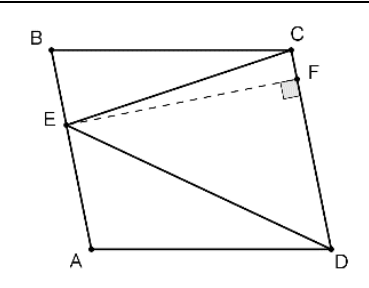

1a

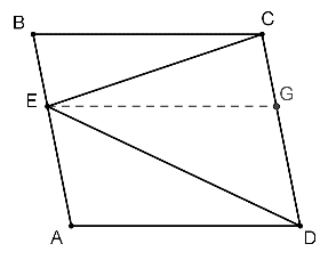

$1 \mathrm{~b}$

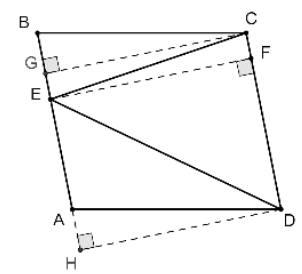

$1 \mathrm{c}$

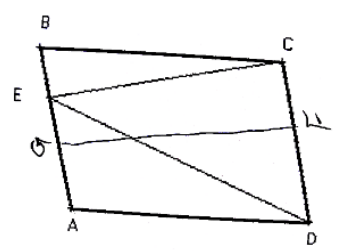

$1 d$

Figure 1. (a-c) Task 1 with auxiliary lines, (d) students' sketch

Task 2: Prove that the quadrilateral formed by the midpoints of the sides of a given quadrilateral is a parallelogram, and that its area is half of the area of the given quadrilateral (Varignon's theorem, Theorem 3.11 in Coxeter \& Greitzer, 1967). Task 2 contains two parts, the second of which relates to area. This two-part structure and the large variety of possible auxiliary lines allowing to make use of properties of a triangle's midsegment and of a parallelogram to prove the claim make it especially suitable for our study (see students' sketches in Figure 2 in the Findings section).

\section{Procedure}

We used two interview techniques to obtain information on the introduction of auxiliary lines: stimulated recall $(\mathrm{SR})$ and partial revealing (PR). SR is a reflective method used in qualitative research (e.g., Bikner-Ahsbahs, 2004) in which students carry out a task undisturbed and are later prompted by the use of some visual or auditory stimulus to recall their thoughts while carrying out the task. A crucial advantage of SR is that it does not interfere with carrying out the task. Thus, we decided to use SR in the relatively simple first task. Also, the SR sessions which followed the first task familiarized students with the researchers' request to reflect upon their actions.

In the more complex Task 2, we wanted to make sure that students do not get stuck. For this purpose, we developed and used the idea of partial revealing (PR), a variation on task-based interviews (Goldin, 2000): We prepared a progressively more revealing sequence of 12 visual or verbal hints. The hints were descriptions/ sketches of an auxiliary line or some key idea/ definition, supporting different ways to prove Task 2 (cf. hints to the same problem in de Villiers, 2014). The hints helped students recalling relevant knowledge or focusing on specific information given in the problem. After obtaining a hint, the students were encouraged to reflect upon the new situation 
created by the hint. This approach helps the students (and the researcher) to understand and describe the possible reasons for introducing an auxiliary line, whether they tried to use a definition, a previously solved problem or just feel that the hint will advance the proof.

In a first meeting (about 20 minutes), each pair of students worked on Task 1 (Figure 1) without researcher intervention. The solution, explanation, or proof, agreed upon by the two students was collected by the researcher without comment. In the second meeting (about $45 \mathrm{~min}$ ), two days after the first one, the student pairs returned to Task 1, using SR. In the third meeting (about 45 min), the students worked together on Task 2 . The PR method was applied.

The qualitative analysis of the data was based on the methodology used by Jones (1998) for the exploration of students' solutions of geometric construction problems on the subject of interplay between students' intuitive and deductive reasoning. First, the video-recordings of all interviews and the students' written productions were searched for auxiliary lines drawn by the students. Then, the videos were transcribed; we read the transcripts of all the meetings line by line, and marked phrases that seemed to relate to reasons for adding auxiliary elements. Next, students' actions and utterances while introducing the auxiliary lines were coded according to our adaptation of Pólya's (1957) list of reasons for introducing of auxiliary lines. For instance, the utterance "The subject of the problem is an area, we need to draw the height" which accompanied introduction of the line segment EF (Figure 1c) was coded as "recalling" (going back to definition/ concretizing a formula). The utterance "It just seems right to draw this line" about the same auxiliary line was coded as "anticipating" (expecting to make the original problem more suggestive). These broad categories of reasons were further divided according to the type of auxiliary line and the type of reason.

When analyzing Task 1, we juxtaposed students' explanations during the SR sessions with their explanations to their partners during the problem-solving meetings and with written productions and sketches. We paid special attention to the general context of the proof and in particular to the change in students' actions and utterances after introducing the auxiliary line; in this, we followed Goldin's (2000) way of analyzing interactions between the subjects and mathematical structures of the tasks. This procedure helped us to confirm and elaborate our initial coding.

When analyzing Task 2 we paid special attention to the moments where specific PR hints (definitions, formulas or visual images) led to the introduction of an auxiliary line; again we followed Goldin (2000), this time with respect to the role of hints in the analysis of students' problem-solving behavior.

\section{Findings}

We analyzed five pairs of students. Here ${ }^{1}$, we present the case of Bob and Boris whose reasons were of both types: "recalling" and "anticipating".

\footnotetext{
${ }^{1}$ Analyses of two more cases are presented elsewhere (Palatnik \& Dreyfus, 2018)
} 


\section{Task 1}

Boris soon drew the auxiliary line GF (Figure 1d) stating: "I want to calculate the area of CED and then the area of the two smaller triangles, to compare." In the SR session, he described GF as a height through the midpoint of $\mathrm{AB}$, and gave the following reasons for the introducing GF: "Because they ask for area, and the height is a part of the area formula" (Bob\&Boris SR10). When asked about the reason for drawing the height exactly at the midpoint of $\mathrm{AB}$, Boris can't give a specific reason: "It was the first point to draw the height"; "it was an initial thought" (Bob\&Boris SR 28, 36). In the SR session Bob recalled that immediately after Boris drew the auxiliary line, he thought that there are probably more places where the area is the same.

The students' solution of Task 1 culminated in the following exchange:

Boris: $\quad$ The height will always be the same!

Bob: Let's assume that I place it, point E, together with point A [Points at the sketch, draws imaginary AC].

Boris: [loud] Look, it is at every point!

Bob: [continues with confidence] So, it happens at every point.

Boris: $\quad$ The base is the same base, and the height is the same height, so at every point it will be equal.

Bob: $\quad$ U-hu, if I place it there, at point A (draws imaginary line AC=EC), it is diagonal, it halves the parallelogram. If I place it at the point $\mathrm{B}$, diagonal $\mathrm{BD}$ also halves the parallelogram.

Bob: $\quad$ So, it looks to me, that at every point. (Bob\&Boris M1 35-41)

Analysis of Task 1. The auxiliary elements introduced by the students in the course of solving the task were: (1) GF - the height through the midpoint of $\mathrm{AB}$; (2) and (3) diagonals $\mathrm{AC}$ and $\mathrm{BD}$ (imagined). As follows from the students' explanation given above, the reason to use height was that it forms part of the formula for the area and thus of recalling nature. A possible explanation for the choosing the height through the midpoint of the side is the intuitive rule same A- same B (Stavy, $\&$ Tirosh, 2000), namely, half of the area (A) - half way on the side (B). As for the diagonals AC and $\mathrm{BD}, \mathrm{Bob}$ imagined them and gestured them in the air. In the SR session, he explained that if $\mathrm{E}$ is placed at the vertices A and B it's easy to see that the diagonals AC and BD halve the parallelogram (anticipating).

\section{Task 2}

The students started by drawing their own sketch (Figure 2a). Boris soon focused on the second part of the task: the area of the parallelogram is half that of the quadrilateral. Bob drew EG, a height in $\mathrm{ABCD}$, as first auxiliary line, but the students realized that they do not know a formula for the area of a general quadrilateral. They received as hints (a) an accurate sketch of the task; (b) a drawing of a triangle with a midsegment. The midsegment immediately triggered progress. Boris drew the diagonals $\mathrm{AC}$ and $\mathrm{BD}$, and Bob formulated a full formal proof to the first part of the task, using the property that a midsegment of a triangle is parallel to the third side of the triangle. 
To prove the second part of the task, the students wanted to calculate areas and looked for a place to construct a height. A switch from calculating to comparing areas was initiated when they attended to pairs of congruent triangles and in particular to the triangles AHN and HDI (Figure 2b, 2c). Bob suggested comparing their areas to that of quadrilateral HIMN, and Boris introduced the crucial auxiliary line NI (Figure 2c).

Boris: I have constructed a diagonal [of HIMN]. And [points to the sketch] this angle equals this, 'cause it is parallel.

Bob: $\quad$ No, you still have to prove this.

Boris: Wait a minute [rotates the sheet with the sketch]. We have to prove that these areas are equal [points to the triangles AHN, HDI, NMI and IHN]. It will make it a midsegment (of triangle AMD) (Bob\&Boris M3 134-140).

Later the students developed this idea into a proof (Figure 2d).

Analysis of Task 2. The main auxiliary elements introduced by the students in the course of task solving were: (1) EG-the segment connecting the midpoints of $\mathrm{AB}$ and $\mathrm{CD}$; (2) diagonals $\mathrm{AC}$ and $\mathrm{BD}$ and (3) NI- the midsegment of the triangle AMD (and the three analogous midsegments in the other parts of the quadrilateral).

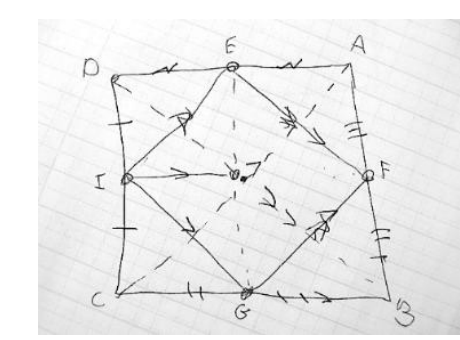

$2 \mathrm{a}$

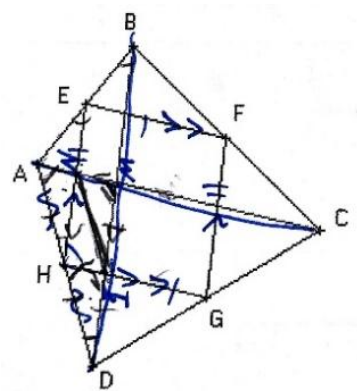

$2 b$

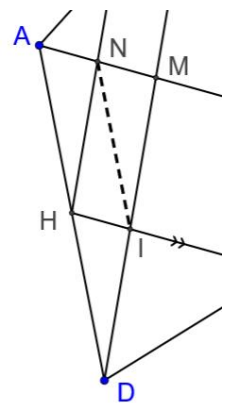

$2 \mathrm{c}$

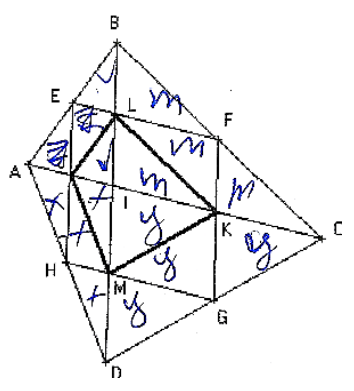

$2 d$

Figure 2. The students' sketches for Task 2 (2c is our copy of a relevant part of students' sketch $2 \mathrm{~b}$ )

We may assume that the auxiliary line EG, perceived by the students as a height, was introduced as a part of a recalled procedure which connects between height as an auxiliary line and height as a part of the area formula as previously mentioned by Boris.

The diagonals AC and BD were introduced immediately after the visual hint (a drawing of a triangle with a midsegment). We assume that the hint led the students to recall the definition and properties of a midsegment, which became a reason for introducing $\mathrm{AC}$ and $\mathrm{BD}$ which in turn helped them elaborate the sketch into a formal proof.

We interpret that the reason for introducing the crucial auxiliary line NI was an anticipating one. Despite the fact that Boris was not sure how this auxiliary line will advance his proof, he introduced it wishing to show that the areas of quadrilateral HIMN and the combined areas of HDI and AHN are equal. It is remarkable that in the collaborative problem-solving efforts of Bob and Boris in Tasks 1 and 2 the reasons to introduce auxiliary lines were both of recalling and anticipating nature. 


\section{Discussion}

Our aim was to reveal and characterize students' reasons for introducing auxiliary lines in geometric proof situations. The five pairs of students who participated in the study introduced a total of 26 auxiliary elements of various nature: heights of triangles and parallelograms, diagonals of parallelograms, triangle midsegments, and medians. Some of these elements were actually drawn, others imagined. As we expected, Task 2 was not easy for students to prove (cf. Ding and Jones, 2006; Hsu and Silver, 2014 about the complexity of tasks with auxiliary lines).

The reasons students gave when introducing auxiliary elements are of two types (1) recalling some known formulas, definitions and procedures, and (2) anticipating to receive more information from a modified situation. There were 14 auxiliary elements of the first type and 12 of the second.

In the tasks we used, most of the reasons of a recalling nature were related to the introduction of heights. The students drew heights as part of a learned algorithm for finding areas: height is explicitly present in the area formula; hence students recalled the formula, and almost immediately drew heights as auxiliary lines; this seems to be a pertinent feature of students' proving process in the context of areas. It was very salient in the interview with Bob and Boris.

Our data allow us to elaborate on characteristics of Pólya's third type of reason for introducing auxiliary lines: anticipating to make problem fuller, more suggestive, without knowing exactly how the auxiliary line will be used. Most of the examples of such reasons we found can be organized into two groups. In the first group (4 occurrences), we put reasons related to intuitive rules (Stavy, $\&$ Tirosh, 2000). Evidence for this is provided by the intuitive "half side-half area" reasoning when introducing the auxiliary lines through the midpoint of the side (e.g., Bob and Boris, Task 1, line GF). In the second group of anticipating reasons for introducing auxiliary lines (6 occurrences), we put reasons related to the students' feeling that they exhausted the available resources and they needed to produce some new source of information (e.g., Bob and Boris, Task 2, NI).

All students used area formulas when proving Task 1; a majority also introduced heights in Task 2, and when this failed, they introduced other auxiliary lines. One possible explanation for this is, that when the students felt that they had exhausted the resources suggested by recall, they tried auxiliary lines based on some anticipation, while keeping in mind that they have to substantiate it (as happened to Bob and Boris when introducing NI in the second part of Task 2).

Based on these findings, we suggest that the introduction of auxiliary lines in proving situations can be expected to provide a rich context for the development of geometrical thinking. Teachers should encourage proactive and reflective approaches to the introduction of auxiliary elements. It is impossible to develop mastery related to the introduction of auxiliary lines without creating experiences of drawing, imagining and dynamically modifying auxiliary lines. This study of students introducing auxiliary elements when proving included only a small number of participants, and only two tasks from a single geometry topic, and it was conducted with paper and pencil only. Further research is required to better understand the complex dynamics of introducing auxiliary lines.

\section{References}


Battista, M. T. (2007). The development of geometric and spatial thinking. In F. Lester (Ed.), Second handbook of research on mathematics teaching and learning (pp. 843-908). Charlotte, NC: NCTM/Information Age.

Bikner-Ahsbahs, A. (2004). Towards the emergence of constructing mathematical meanings. In M. J. Høines \& A. B. Fuglestad (Eds.), Proceedings of the $28^{\text {th }}$ Annual Conference of the International Group for Psychology of Mathematics Education, Vol. 2 (pp. 119-126). Bergen, Norway: Bergen University College.

Coxeter, H. S. M. \& Greitzer, S. L. (1967). Geometry revisited. Washington, D.C.: Mathematical Association of America.

De Villiers, M. (2014). Slaying a geometrical monster: Finding the area of a crossed quadrilateral. The Scottish Mathematical Council Journal, 44, 71-74.

Ding, L., \& Jones, K. (2006). Teaching geometry in lower secondary school in Shanghai, China. Proceedings of the British Society for Research into Learning Mathematics, 26, 41-46.

Fan, L., Qi, C., Liu, X., Wang, Y., \& Lin, M. (2017). Does a transformation approach improve students' ability in constructing auxiliary lines for solving geometric problems? An interventionbased study with two Chinese classrooms. Educational Studies in Mathematics, 96, 229-248.

Fujita, T., \& Jones, K. (2014). Reasoning-and-proving in geometry in school mathematics textbooks in Japan. International Journal of Educational Research, 64, 81-91.

Goldin, G. A. (2000). A scientific perspective on structured, task-based interviews in mathematics education research. In A. Kelly \& R. A. Lesh (Eds.), Handbook of research design in mathematics and science education (pp. 517-545). Mahwah, NJ: LEA.

Herbst, P. (2004). Interactions with diagrams and the making of reasoned conjectures in geometry. ZDM - The International Journal on Mathematics Education, 36, 129-139.

Herbst, P., \& Brach, C. (2006). Proving and doing proofs in high school geometry classes: What is it that is going on for students? Cognition and Instruction, 24, 73-122.

Hsu, H. (2007). Geometric calculations are more than calculations. In J.-H. Woo, H.-C. Lew, K.-S. Park, D.-Y. Seo (Eds.), Proceedings of the 31st Conference of the International Group for the Psychology of Mathematics Education, Vol. 3 (pp. 57-64). Seoul, Korea.

Hsu, H. Y., \& Silver, E. A. (2014). Cognitive complexity of mathematics instructional tasks in a Taiwanese classroom: An examination of task sources. Journal for Research in Mathematics Education, 45, 460-496.

Jones, K. (1998), Deductive and intuitive approaches to solving geometrical problems. In: C. Mammana \& V. Villani (Eds.), Perspectives on the teaching of geometry for the 21st century (pp. 78-83). Dordrecht, The Netherlands: Kluwer.

Miyazaki, M., Fujita, T., \& Jones, K. (2015). Flow-chart proofs with open problems as scaffolds for learning about geometrical proofs. ZDM - The International Journal on Mathematics Education, 47, 1211-1224.

Palatnik, A., \& Dreyfus, T. (2018). Students' reasons for introducing auxiliary lines in proving situations. The Journal of Mathematical Behavior. https://doi.org/10.1016/j.jmathb.2018.10.004.

Pólya, G. (1957). How to solve it. $2^{\text {nd }}$ edition. Princeton, NJ: Princeton University Press.

Schoenfeld, A. H (2000). Purposes and methods of research in mathematics education. Notices of 
the American Mathematical Society, 47, 641-649.

Stavy, R., \& Tirosh, D. (2000). How students (mis)understand science and mathematics: Intuitive rules. New York: Teachers College Press.

Senk, S. L. (1985). How well do students write geometry proofs? The Mathematics Teacher, 78, 448-456. 